\section{D.002 DELAYS AND DETERMINANTS OF TRAUMATIC BRAIN INJURY CARE OUTCOME IN LOW-INCOME UGANDA}

1,2Scholar Herman Lule*, ${ }^{3}$ Mbaaga Ssebakumba, ${ }^{4}$ Francis Olweny, ${ }^{5}$ Jacquiline Mabweijano, ${ }^{6}$ Joel Kiryabwire. ${ }^{1}$ Department of Surgery, Kampala International University P.O.Box 71, Bushenyi, Uganda; ${ }^{2}$ Injury Epidemiology and Prevention (IEP) Research Group; P.O.Box 52; Fl-20521 Turku, Turku Brain Injury Centre, Turku University Hospital and University of Turku, Finland; ${ }^{3}$ Department of General Surgery, School of Medicine, College of Health Sciences, Makerere University, P.o.Box 7072, Kampala, Uganda; ${ }^{4}$ Clinical Epidemiology Unit, School of Medicine, College of Health Sciences, Makerere University, Kampala, Uganda; ${ }^{5}$ Department of Causality, Trauma and Emergency Medicine, Mulago National Referral and Teaching Hospital, P.o.Box 7051, Kampala, Uganda; ${ }^{6}$ Department of Neurosurgery, Mulago National Referral Hospital and Teaching Hospital, P.o.Box 7051, Kampala, Uganda

\subsection{6/injuryprev-2021-safety.78}

Background Increasing traumatic brain injury (TBI) has paralleled need for decompression surgery for acute subdural $(\mathrm{ASDH})$ and acute extradural haematoma (AEDH). Knowledge of key determinants of clinical outcomes inclusive of delays is mandatory to guide treatment protocols.

Objective To determine the thirty-day clinical outcomes and predictor variables for patients with extra-axial haematoma at Mulago National Referral Hospital in Uganda.

Methods Prospective observational cohort study of 109 patients with Computed Tomography (CT) confirmed extraaxial haematoma. Ethical Clearance was obtained from School of Medicine Research and Ethics Committee of Makerere University (REC REF. 2018-185). Admitted patients were followed-up for Glasgow Outcome Scale (GOS) and final disposition. Multivariate regression analysis was performed using Stata 14.0 (StataCorp. 2015) at 95\% confidence-interval, regarding $\mathrm{p}<0.05$ as statistically significant.

Results No participant received surgery within six hours of decision making. The overall proportion of favourable outcome was $71.7 \% \quad(n=71)$, with $42.3 \% \quad(n=11)$ and $81.7 \%$ $(\mathrm{n}=58)$ for ASDH and AEDH respectively $(\mathrm{p}=0.111)$. Factors associated with a favourable outcome were: Admission Systolic $\mathrm{BP}>90 \mathrm{mmHg} \quad[\mathrm{IRR}=0.88 \quad(0.26-0.94) \quad 95 \% \mathrm{CI}, \quad \mathrm{p}=0.032)$, Oxygen saturation $>90 \% \quad[$ IRR $=0.5 \quad(0.26-0.94) \quad 95 \% \mathrm{CI}$, $\mathrm{p}=0.030]$ and Diagnosis AEDH $[\mathrm{IRR}=0.53(0.30-0.92) 95 \%$ $\mathrm{CI}, \mathrm{p}=0.025)$. Moderate $[\mathrm{IRR}=4.57 \quad(1.15-18.06) \quad 95 \% \mathrm{CI}$, $\mathrm{P}=0.03]$ and severe TBI $[\mathrm{IRR}=6.79 \quad(2.32-19.86) \quad 95 \% \mathrm{CI}$, $\mathrm{p}<0.001]$ were associated with unfavorable outcomes.

Conclusion The study revealed that amidst delays, post resuscitation GCS, Systolic BP, Oxygen circulation and diagnosis of $\mathrm{AEDH}$ at admission, are the most important determinants of outcome for patients with extra-axial intracranial haematoma. These findings are valuable for triaging teams in resource constrained settings.

\section{D.003 ASSESSMENT OF TRAUMA PATIENTS FOR THE DEVELOPMENT OF TRAUMA PROTOCOL IN NEPAL}

Santosh Paudel ${ }^{*}$, Siddhartha Dhungana. National Trauma Center, Kathmandu, Nepal

\subsection{6/injuryprev-2021-safety.79}

Background National Trauma Center is the only specialized trauma care hospital in Nepal in operation since 2012. In absence of a standard, trauma care protocol, the patients attending the hospital are separated by a team of medical officer and nurses at Emergency Department into four colourcoded triage categories of Red, Amber, Green and Black. This study contributes to the development of a standard trauma protocol by assessing the injuries and outcomes of the patients.

Methods We included all patients presented Emergency Department with at least one trauma during Jan 2019 to Dec 2019. Patients Demographic details, vitals, laboratory tests, radio imaging records and clinical examination were entered in hospital software and stored in the hospital database. The data were extracted from the database with the permission of the hospital authority.

Results Total of 18,985 patients was presented in the emergency department with different types of injury. Among them, 2,663 (14.0\%) were needed special care and treated in inpatient wards. Fall and road crash comprised almost $80 \%$ of admitted cases. Mortality was $2.1 \%$ of admitted patients, mostly with head injuries followed by multiple injuries.

Conclusion Disproportionately high cases of fall and road crash warrant a standard protocol for treating them. The proportion of death is also significant which indicates a need for ambulatory Emergency Medical Services in order to stabilise patients.

Learning Outcomes If there were a standard protocol for trauma management mortality could be minimised.

\section{D.004 PROCESS EVALUATION OF A CONCUSSION-RELATED POPULAR OPINION LEADER INTERVENTION}

Zachary Kerr*, Paula Gildner, Stephanie Krieg, Johna Register-Mihalik. University of North Carolina At Chapel Hill, Chapel Hill, USA

\subsection{6/injuryprev-2021-safety. 80}

Background A Popular Opinion Leader (POL) educational intervention was developed/implemented to improve concussion management in middle school (MS) sports. Parents/ coaches/staff recruited as POLs received three online sessions including concussion education and concussion prevention/ management conversation skills development. We assessed POLs' evaluations of the sessions and their resulting concussion-related conversations.

Methods The intervention occurred within seven MS in the Southeastern United States. MS parents/coaches/staff were recruited to participate in the training sessions (22 parents and 14 coaches/staff). One-month post-training, POLs completed an online questionnaire and/or in-person interview data evaluating their training and conversations. Quantitative data provided conversation frequencies and topic; qualitative data identified pertinent themes using template analysis.

Results Fifteen (41\%; 7 parents, 8 coaches/staff) and six (17\%; 1 parent, 5 coaches/staff) POLs completed questionnaires and interviews, respectively. Forty-one conversations were reported (average 2.6 per person). POLs commonly reported talking with their children (47\%), spouse/partner (40\%), and teachers/staff (33\%); common discussion topics included concussion signs/symptoms (73\%), responding to suspected concussion (67\%), and playing safe to reduce concussion risk (60\%). POL interviews identified trainings as concise, yet thorough. Conversations were initiated when potential concussions occurred during games. POLs felt knowledgeable and found people receptive to information. Talking to those with previous concussion experience was easier. POLs suggested additional trainings should provide easily shareable concussion recognition resources.

Conclusion POLs responded well to training session materials. Identified areas of improvement, such as providing shareable 